\title{
Neonatal Aspects: Is There Continuity?
}

\section{Milan Stanojevic}

\section{ABSTRACT}

During the 9 months between conception and birth, the fetal brain is transformed from instructions in genes to a complex, highly differentiated organ. The human central nervous system (CNS) changes from a microscopic band of embryonic neuroblasts to a $350 \mathrm{gm}$ mass with more than 109 interconnected highly differentiated neurons in the cortex alone. How this extraordinary growth results in sensomotor, cognitive, affective and behavioral development is still unexplored. The development of voluntary, cognitive and purposive activity from fetal to neonatal period is to analyze the developmental transformations of the brain expressed by development of movement patterns from prenatal through postnatal period. As the development of the brain is unique and continuing process throughout the gestation and after birth, it is expected that there is also continuity of fetal to neonatal movements which are the best functional indicator of developmental processes of the brain. Concerning the complexity, voluntary control and stereotype, there are at least four groups of movements: Reflexes, fixed action patterns, rhythmic motor patterns, and directed movements. Substantial indications suggest that spontaneous activity is a more sensitive indicator of brain dysfunction than reactivity to sensory stimuli in reflex testing. It was proved that assessment of general movements in high-risk newborns has significantly higher predictive value for later neurological development than neurological examination.

Nutritional stress at critical times during fetal development can have persistent and potentially irreversible effects on organ function. Impaired intrauterine growth and development may antecede insufficient postnatal growth. Thus, it may be a marker of impaired central nervous system integrity because of adverse intrauterine conditions. Unfavorable intrauterine environment can affect adversely fetal growth. There is an association between postnatal growth and neurodevelopmental outcome.

Concerning the continuity from fetus to neonate in terms of neurobehavior, it could be concluded that fetus and neonate are the same persons in different environment. While in the womb, fetus is protected from the gravity which is not so important for its neurodevelopment, postnatally the neonate is exposed to the gravity during the labor and from the first moments of autonomous life. Development of motor control is highly dependent on antigravity forces enabling erect posture of infant or young child. These environmental differences should be kept on mind during prenatal as well as postnatal assessment.

Keywords: Neurobehavior, Fetus, Neonate, 4D ultrasound, General movements.

How to cite this article: Stanojevic M. Neonatal Aspects: Is There Continuity? Donald School J Ultrasound Obstet Gynecol 2012;6(2):189-196.

Source of support: Nil

Conflict of interest: None declared

\section{INTRODUCTION}

Neurobehavior is expression of development of central nervous system (CNS) (in particular the brain), which is complex ongoing process throughout gestation and after birth. ${ }^{1-3}$ It is important to understand how CNS produces different kind of movements and which of them are important for the assessment of disturbed CNS development. Concerning the complexity, voluntary control and stereotypy, there are at least four groups of movements: Reflexes, fixed action patterns, rhythmic motor patterns, and directed movements. ${ }^{1-3}$ Reflexes (tendon, papillary) are the simplest involuntary, stereotyped and graded responses to sensory input, and have no threshold except that the stimulus must be great enough to activate the relevant sensory input pathway. ${ }^{1-3}$ Fixed action patterns (sneezing, orgasm) are involuntary and stereotyped, but typically have a stimulus threshold that must be reached before they are triggered, and are less graded and more complex than reflexes. Rhythmic motor patterns (walking, breathing) are stereotyped and complex, but are subject to continuous voluntary control. ${ }^{1-3}$ Directed movements (reaching) are voluntary and complex, but are generally neither stereotyped nor repetitive. Rhythmic motor patterns are complex (unlike reflexes) yet stereotyped (unlike directed movements) and, by definition, repetitive (unlike fixed action patterns). ${ }^{1-3}$ It is presumed that the basic rhythmicity and patterning of rhythmic motor patterns are produced by neural networks termed central pattern generators. ${ }^{4}$ Fetuses and newborns exhibit a large number of endogenously generated motor patterns, which are presumably produced by central pattern generators located in different parts of the brain. ${ }^{4}$ Moreover, substantial indications suggest that spontaneous activity is a more sensitive indicator of brain dysfunction than reactivity to sensory stimuli in reflex testing. ${ }^{4}$ It has been demonstrated that in newborn infants affected by different brain lesions, spontaneous motility does not change in quantity, but it loses its elegance, fluency and complexity. ${ }^{5}$ As the development of the brain is unique and continuing process throughout the gestation and after birth, it is expected that there is also continuity of fetal to neonatal movements which are the best functional indicator of developmental processes of the brain. ${ }^{1-3}$

The aim is to present possible factors which may influence the continuity of neurobehavior from prenatal to postnatal life. 


\section{Growth and Brain}

The answer to the eternal question concerning the beginning of human life is not simple and unequivocal. It seams that the moment of fecundation is the beginning of new life having the unique potential for development and growth. Prenatal and postnatal potential for growth of the human being is different, with the tendency of slowing down after birth. It seems like potential for growth differs pre- and postnatally, which is controversial. ${ }^{6}$

It could be speculated that development of the morphology and the function should be in equilibrium during different developmental stages. ${ }^{6}$ It is known that prenatal and postnatal growth potential of the heart, liver, kidneys and lungs is different compared to the brain (Figs 1 and 2). ${ }^{7}$

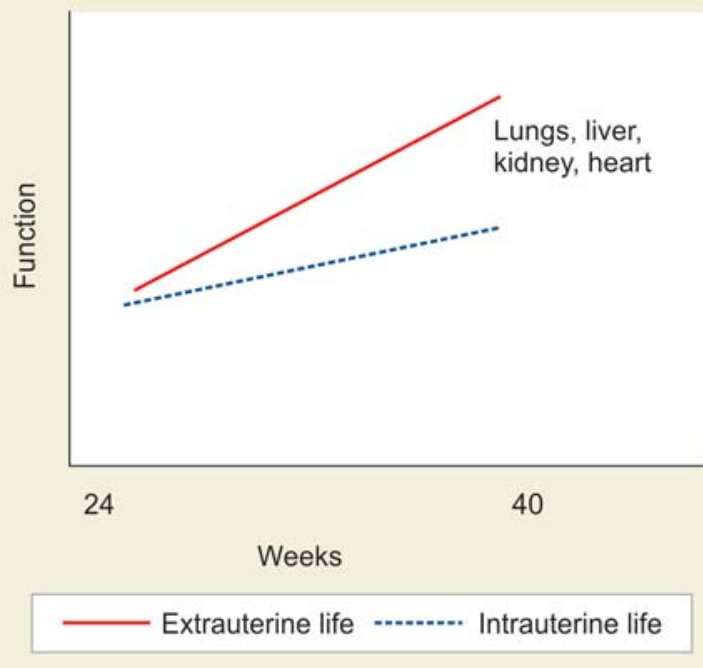

Fig. 1: Potential for prenatal and postnatal growth and function of lungs, liver, kidney and heart

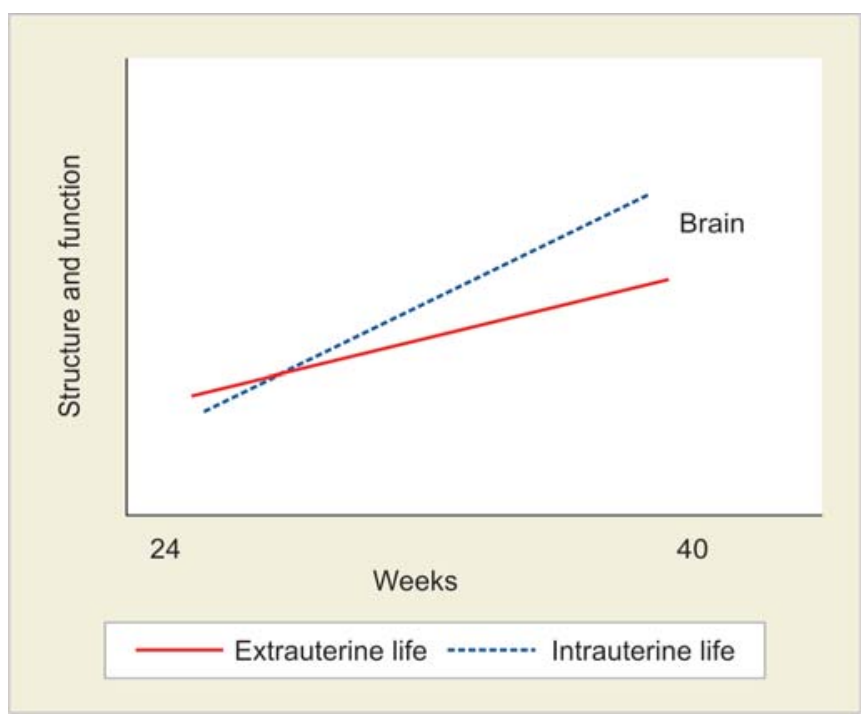

Fig. 2: Potential for prenatal and postnatal brain growth and function
The result of the brain development and growth is the mature brain. ${ }^{8}$ According to the recent data, it is estimated that mature human brain has 86 billion neurons in total and 85 billion of nonneurons. ${ }^{8}$ Cerebral cortex size is $82 \%$ of the brain mass with 16 billion neurons which is $19 \%$ of total brain neurons. ${ }^{8}$ Among primates, humans enjoy the largest number of neurons from which to derive cognition and behavior as a whole. ${ }^{8}$ Neocortex, a new and rapidly evolving brain structure in mammals, has a similar layered architecture in species over a wide range of brain sizes. ${ }^{8}$ Larger brains require longer fibers to communicate between distant cortical areas; the volume of the white matter that contains long axons increases disproportionally faster than the volume of the gray matter that contains cell bodies, dendrites, and axons for local information processing. ${ }^{9}$

Cortical growth is achieved predominantly by an increase in surface area rather than thickness, and during late fetal human development a rapid increase in brain size occurs with considerable development of cortical surface area relative to cerebral volume, manifested in the development of cortical convolutions. ${ }^{10}$

\section{Danger of Adverse Intrauterine Conditions on Fetal Growth}

Nutritional stress at critical times during fetal development can have persistent and potentially irreversible effects on organ function. ${ }^{11}$ Unfavorable intrauterine environment can affect adversely fetal growth. It was shown in one study that fetuses delivered at term from pregnancies complicated by threatened preterm labor were relatively smaller than their peers at birth compared to that at the end of the second trimester (Fig. 3). ${ }^{12}$

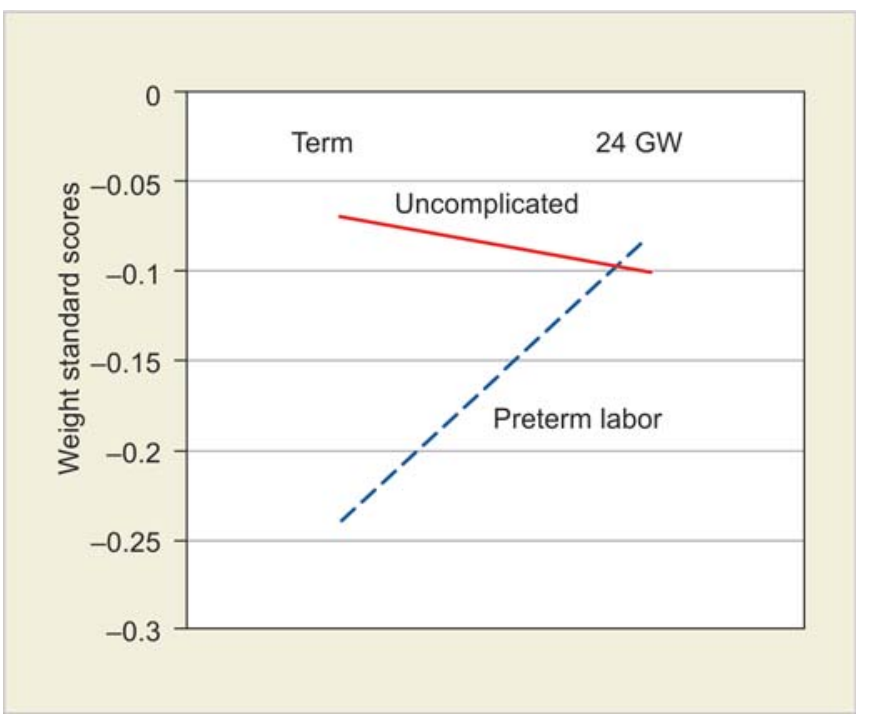

Fig. 3: The fetuses delivered at term from pregnancies complicated by preterm labor were relatively smaller compared to their peers at birth than they had been at the end of the second trimester ${ }^{13}$ 
Spontaneous preterm labor carries significant health consequences in terms of neonatal morbidity and mortality as it is responsible for about $45 \%$ of preterm births, which are approximately 5 to $12 \%$ of pregnancies presently in developed countries. ${ }^{12}$ Preterm labor is one of the great obstetrical syndromes, and while different etiologies, including vascular disease, infection or inflammation, stress and uterine overdistension may contribute to its onset, in many cases, no specific etiology has been identified to date. ${ }^{12}$

There are at least three reasons why neonates born at term after an episode of preterm labor are more likely to be small relative to their peers. First, it is possible that the 'preterm labor event' itself constitutes an insult to fetal growth, altering the subsequent fetal growth rate and trajectory. ${ }^{12}$ Second, it is possible that an altered fetal growth pattern contributes to and eventually culminates in the phenotype of preterm labor. ${ }^{12}$ Third, it is possible that one of the underlying causes of preterm labor provokes both the event and the subsequent growth insult. ${ }^{12}$ Analysis identified that a greater proportion of fetuses delivered at term after an episode of preterm labor experienced downward centile crossing during the third trimester compared to those from uncomplicated pregnancies. ${ }^{12}$ Fetuses from pregnancies complicated by preterm labor were $43 \%$ less likely to experience upward centile crossing than their peers from uncomplicated pregnancies. ${ }^{12}$

In very low birth weight infants (VLBW) children, the course of postnatal growth rather than the appropriateness of weight for gestational age at birth determines later neurodevelopmental outcome. ${ }^{13}$

The most striking result of that study was postnatal catch-down growth in a group of appropriate for gestational age (AGA) VLBW resulting in weight below the 10th percentile at age $2 .{ }^{13}$

In the human, intrauterine growth retardation (IUGR) can result in persistent postnatal growth failure, which may be attributable, in part, to abnormal growth hormone (GH) secretion. ${ }^{11}$ Whether putative alterations in GH secretion are the result of abnormalities intrinsic to the pituitary or reflect changes in the production of GH-releasing hormone or somatostatin is unknown. ${ }^{11}$ The hypothesis was tested that growth failure associated with IUGR or early postnatal food restriction is caused by a central defect in hypothalamic somatostatin gene expression. ${ }^{11}$ These observations suggest that nutritional stress during a critical developmental window produces a persistent and potentially irreversible alteration in the activity of the hypothalamic-pituitary axis. ${ }^{11}$

\section{Growth Impairment and Neurodevelopmental Outcome}

Weight, length and head circumference at birth were not significantly associated with neurodevelopmental outcome at age 2 for small for gestational age (SGA) or AGA VLBW children. ${ }^{13}$ However, weight and length at age 2 correlated with psychomotor developmental index (PDI) in SGA and AGA children. ${ }^{13}$ These findings indicated an association between postnatal growth and neurodevelopmental outcome. $^{13}$

Different postnatal growth patterns were significantly associated with neurodevelopmental outcome. SGA children who showed substantial catch-up growth with weight above the 10th percentile at age two had neurodevelopmental outcomes comparable to AGA children whose weight remained appropriate for age; whereas SGA children who remained below the 10th percentile by age 2 were impaired in their motor development. ${ }^{13}$ Poor catch-up growth was more frequently associated with motor, but less so with mental delay. ${ }^{13}$ Their mental and motor functioning was significantly poorer than for AGA children with weight above the 10th percentile at age 2 , and even worse than for SGA children with insufficient catch-up growth. ${ }^{13}$ Thus, AGA children with catch down growth had the highest risk for mental retardation, motor delay and cerebral palsy (CP) among all VLBW children. ${ }^{13}$ These findings were mostly independent of the diagnosis of CP. ${ }^{13}$

The origin of CP in children born at term was considered to be prenatal in $38 \%$, peri/neonatal in 35\% and unclassifiable in $27 \%$, while in children born preterm it was $17 \%$, $49 \%$ and $33 \%$ respectively. ${ }^{14,15}$

Compared with delivery at 40 weeks, prevalence of CP at 37 weeks was 1.91/1000 (95\% CI, 1.58-2.25) and RR was 1.9 (95\% CI, 1.6-2.4); the prevalence at 38 weeks was $1.25 /$ 1000 (95\% CI, 1.07-1.42) and RR was 1.3 (95\% CI, 1.1-1.6); the prevalence of CP at 42 weeks was 1.36/1000 (95\% CI, 1.19-1.53) and RR was 1.4 (95\% CI, 1.2-1.6); and after 42 weeks, the prevalence was 1.44 (95\% CI, 1.15-1.72) with RR of 1.4 (95\% CI, 1.1-1.8). ${ }^{16}$

Impaired intrauterine growth and development may antecede insufficient postnatal growth. Thus, it may be a marker of impaired CNS integrity because of adverse intrauterine conditions. ${ }^{13}$ Children who were AGA at birth, but became underweight at age 2 may have already been on a catch-down curve before birth. ${ }^{13}$ Their postnatal growth course could be the continuation of insufficient intrauterine growth. If pregnancy would have continued until term, those infants may have been born SGA. ${ }^{13}$ Insufficient postnatal growth likely reflects impaired intrauterine development. In the future, this hypothesis could be substantiated by repeated intrauterine ultrasonographic measurements demonstrating relative catch-down of growth variables that persist into postnatal life. ${ }^{13}$ 
Brain development appears to be affected by premature termination of the intrauterine environment following preterm birth. ${ }^{10}$ Half of all surviving infants born at 25 weeks or less show neurodevelopmental impairment at 30 months of age. ${ }^{10}$ At age 6 years, about $40 \%$ have cognitive impairment compared to their classroom peers, and impairments are more severe in boys than girls. ${ }^{10}$ Even among less immature infants over one-third develop neurocognitive and behavioral problems. ${ }^{10}$ This neurocognitive impairment is more severe with earlier gestation and longer exposure to the premature extrauterine environment, suggesting that there is a dose- and gender-dependent effect of prematurity on brain development. ${ }^{10}$

Poor postnatal growth in preterm infants, especially of the head, is associated with increased levels of motor and cognitive impairment at 7 years of age. ${ }^{17}$ This growth restriction appears to occur largely in the postnatal rather than antenatal period and may be amenable to intervention and subsequent improvement in outcome. ${ }^{17}$

How abnormalities of size for gestation are associated with risk of CP is shown in Table $1 .{ }^{18}$

The final diagnosis of CP can be mostly established at the age of 3 years, although even in the earlier period of life the infant may develop different abnormal neurodevelopmental signs which could indicate the development of $\mathrm{CP} .{ }^{19-21}$
However, sometimes even quite severe neurologic abnormalities in the first year or so of life can disappear. ${ }^{19-21}$ In 50\% of children described as neurologically abnormal at age 1 year were neurologically normal at the age of 3 years. ${ }^{19-21}$ Early neuromotor findings should suggest that the infant is in a highrisk category, but they do not indicate that infant has $\mathrm{CP} .{ }^{19-22}$ The diagnosis of $\mathrm{CP}$ should be assigned very cautiously before the age of 24 months, unless the cerebral damage is extremely severe. ${ }^{19-21}$

\section{Volumetric Studies}

In comparison to the term-born infants, the premature infants at term demonstrated prominent reductions in cerebral cortical gray matter volume and in deep nuclear gray matter volume and an increase in cerebrospinal fluid volume. ${ }^{23}$ The major predictors of altered cerebral volumes were gestational age at birth and the presence of cerebral white matter injury. ${ }^{23}$

In a large prospective longitudinal cohort study of 202 preterm and 36 term infants, magnetic resonance (MR) scans at term equivalent were undertaken for volumetric estimates of cortical and deep nuclear grey matter, unmyelinated and myelinated white matter (WM) and cerebrospinal fluid (CSF) within eight parcellated regions for each hemisphere of the brain. ${ }^{24}$ Perinatal correlates analyzed in relation to

Table 1: Risk factors associated with abnormalities of size for gestation in term and near-term infants and their association with risk of cerebral palsy $(\mathrm{CP})^{18}$

\begin{tabular}{|c|c|c|}
\hline $\begin{array}{l}\text { Factor associated with } \\
\text { aberrant size at birth }\end{array}$ & $\begin{array}{l}\text { Is it a risk factor for } \\
\text { cerebral palsy }(C P)\end{array}$ & Is risk of CP primarily present in LGA/SGA? \\
\hline Congenital anomalies $^{a}$ & Yes & No \\
\hline TORCH infections & Yes & No evidence \\
\hline Chromosomal defects & Yes & Not known (NK) \\
\hline Twinning in the $3 r d$ trimester & Yes & $\begin{array}{l}\text { Possibly in very preterm birth } \\
\text { (<32 weeks of gestation)/LGA }\end{array}$ \\
\hline Placental $^{\mathrm{a}}$ and cord anomalies & Yes & No evidence \\
\hline Preeclamptic toxemia ${ }^{a}$ & Yes & No \\
\hline Bacterial genital tract infection & Yes & No evidence \\
\hline Preterm birth ${ }^{\mathrm{a}}$ & Yes & ?No \\
\hline Maternal starvation & Yes & \\
\hline Maternal alcohol abuse & Yes & NK \\
\hline Maternal smoking & NK & \\
\hline Maternal lung/cyanotic heart disease & NK & \\
\hline Maternal renal/malabsorption disease & NK & \\
\hline Maternal diabetes (including gestational) ${ }^{a}$ & ?Yes & NK \\
\hline Small maternal size or low birth weight & NK & \\
\hline Socioeconomic deprivation & Yes & No \\
\hline Neonatal hypothermia & Yes & Little or no contribution \\
\hline Neonatal hypoglycemia & Yes & Little or no contribution \\
\hline Intrapartum stress & Yes & No \\
\hline Clinical signs of birth asphyxia/hypoxia & Yes & No evidence \\
\hline
\end{tabular}

andicates a risk factor also associated with large size at birth. Otherwise these are all risk factors for small size

Abbreviations: TORCH: Toxoplasmosis, other infections, rubella, cytomegalovirus, herpes simplex; LGA: Large for gestational age; SGA: Small for gestational age; CP: Cerebral palsy; NK: Not known. 
regional brain structure included gender, gestational age, intrauterine growth restriction, bronchopulmonary dysplasia, white matter injury (WMI) and intraventricular hemorrhage. ${ }^{24}$ Results revealed region-specific reductions in brain volumes in preterm infants compared with term controls in the parieto-occipital, sensorimotor, orbitofrontal and premotor regions. ${ }^{24}$ Within the sensorimotor and orbitofrontal regions cortical grey matter and unmyelinated WM were most clearly reduced in preterm infants, whereas deep nuclear grey matter was reduced mainly within the parieto-occipital and subgenual regions. CSF (ventricular and extracerebral) was doubled in volume within the superior regions in preterm infants compared with term controls. $^{24}$

Severe regional disruptions to cerebral development were found in preterm infants by term equivalent, which vary in relation to perinatal exposures. ${ }^{24} \mathrm{WM}$ injury (WMI) has the most significant impact together with intrauterine growth restriction (IUGR), influencing more posterior cerebral structures. ${ }^{24}$ BPD has a global impact. ${ }^{24}$

The rates of brain growth are highest in the last part of gestation and the first 1 year of life. ${ }^{25}$ There is evidence that impaired brain growth in utero and in infancy may lead to poorer cognitive function in childhood. ${ }^{25}$ The brain volume a child achieves by the age of 1 year helps determine later intelligence. Growth in brain volume after infancy may not compensate for poorer earlier growth. ${ }^{25}$ However, whether these associations reflect the cumulative effect of brain growth achieved by that age or the influence of critical periods of growth earlier in life has been unclear. ${ }^{25}$ Associations between head circumference and intelligence in later childhood owe more to the influence of brain growth during infancy than they do to growth after infancy. ${ }^{25}$

Extremely immature ( $<26$ weeks) children showed a marked drop in weight in the neonatal period, and continued to decline up to 3 months' corrected age. ${ }^{26}$ After 3 months' corrected age, their weight began to increase and continued to do so, reaching the mean of the reference at 11 years of age. $^{26}$ The mean difference in weight between the extremely immature and control participants was significant at all ages with decreasing tendency toward the 11th year of life. ${ }^{26}$ The proportion of extremely immature children with subnormal weight increased from $7 \%$ at birth to $60 \%$ at 3 months' corrected age, after which there was a reduction in this proportion at later ages. ${ }^{26}$ At expected date of delivery, the mean head circumferences (HC) in extremely immature children were significantly lower than those in their controls. ${ }^{26}$ These remained significantly lower than those in the controls and in the reference mean at all ages at which comparison was possible (Fig. 4) ${ }^{26}$ A significantly higher proportion of the extremely immature cohort

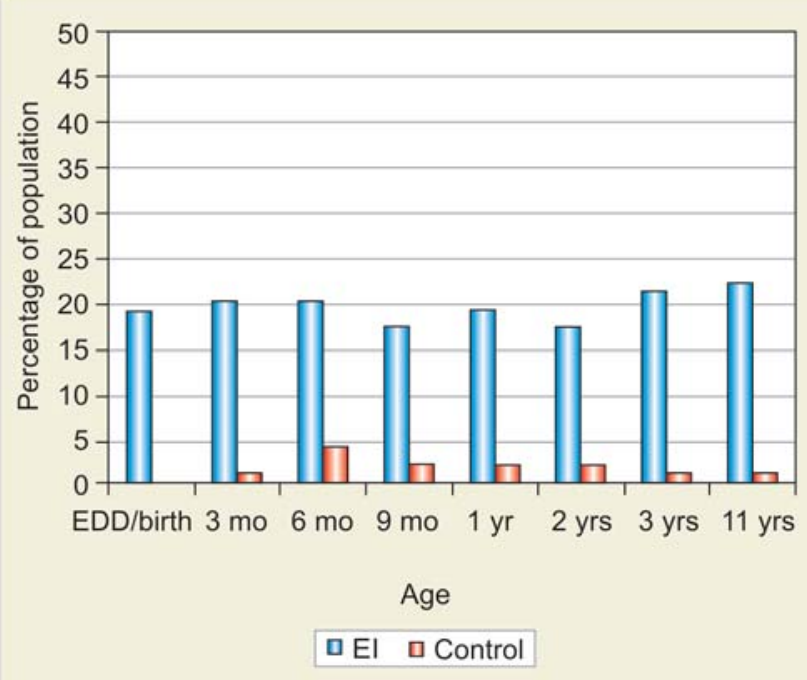

Fig. 4: Percentage of extremely immature (EI) $(<26$ week's gestation) children with subnormal head circumference for age at different ages ${ }^{26}$

compared with controls had subnormal $\mathrm{HC}$ at 11 years of age. In addition, there were significant differences between EI and control children by gender at 11 years of age: The mean $\mathrm{HC}$ of extremely immature boys was $2 \mathrm{~cm}$ lower than that of their counterparts, and it was $1.2 \mathrm{~cm}$ lower in extremely immature girls than in their controls. ${ }^{26}$

In contrast to weight and height, extremely immature children did not exhibit catch-up growth in head circumference after the first 6 months of life. ${ }^{26}$ It means that extremely immature children have growth failure in early extrauterine life in comparison with those of normal intrauterine growth during the third trimester. ${ }^{26}$ The relative decline in growth parameters continued up to 3 months corrected age. ${ }^{26}$

\section{Assessment of General Movements from Prenatal to Postnatal Life}

Heinz Prechtl's work enabled that spontaneous motility during human development has been brought into focus of interest of many perinatologists prenatally and developmental neourologists postnatally. ${ }^{27,28}$ According to the research preceding Prechtl's ingenious idea, during the development of the individual the functional repertoire of the developing neural structure must meet the requirements of the organism and its environment. ${ }^{27,28}$ This concept of ontogenetic adaptation fits excellently to the development of human organism, which is during each developmental stage adapted to the internal and external requirements. ${ }^{27,28}$ Prechtl stated that spontaneous motility, as the expression of spontaneous neural activity, is a marker of brain proper or disturbed function. ${ }^{27,28}$ The observation of unstimulated fetus or infant which is the result of spontaneous behavior without sensory stimulation is the best method to assess its 
central nervous system capacity. ${ }^{27,28}$ All endogenously generated movement patterns from unstimulated central nervous system could be observed as early as from the 7 to 8 weeks of postmenstrual age, with developing a reach repertoire of movements within the next 2 or 3 weeks, continuing to be present for 5 to 6 months postnatally. ${ }^{27,28}$ This remarkable fact of the continuity of endogenously generated activity from prenatal to postnatal life is the great opportunity to find out those high-risk fetuses and infants in whom development of neurological impairment is emerging. ${ }^{27,28}$ The most important among those movements are so called general movements (GMs) involving the whole body in a variable sequence of arm, leg, neck and trunk movements, with gradual beginning and the end. ${ }^{27,28}$ They wax and wane in intensity, force and speed being fluent and elegant with the impression of complexity and variability. ${ }^{27,28} \mathrm{GMs}$ are called fetal or preterm from 28 to 36 to 38 weeks of postmenstrual age, while after that we have at least two types of movements: Writhing present to 46 to 52 weeks of postmenstrual age and fidgety movements present till 54 to 58 weeks of postmenstrual age. ${ }^{27,28}$ Lack of fluency and existence of considerable variation and complexity are the main characteristics of mildly abnormal GMs. ${ }^{28}$ When complexity, variation and fluency are absent, than we are dealing with definitely abnormal GMs. ${ }^{28}$ The quality of each individual movement includes speed, amplitude and force combined in one complex perception. ${ }^{27,28}$ Some facts are very important in the assessment of GMs. The first is that evaluation of GMs should be based on the video-recorded movements either pre- or postnatally. ${ }^{27,28}$ The second fact is that when assessing GMs one should use so called 'Gestalt perception', which could be described as overall impression of GMs with standardized procedure. ${ }^{27,28}$ During the perception one should recognize the movement patterns of GMs, than assess their complexity, variability and fluency. ${ }^{27,28}$ According to Hadders-Algra, GMs could be classified as normal-optimal, normal-suboptimal, mildly abnormal and definitely abnormal. ${ }^{28}$

It seems that assessment of the quality of GM is a window for early detection of children at high risk for developmental disorders. ${ }^{27,28}$ Method is simple and it is based on so called 'Gestalt perception', i.e. evaluation of GM complexity, variation and amplitude. ${ }^{27,28}$ Assessment of GMs at 2 to 4 months postterm at so called fidgety GM age has been found to have the highest predictive value for development of CP, if abnormal. ${ }^{27,28}$

It was proved that assessment of GMs in high-risk newborns has significantly higher predictive value for later neurological development than neurological examination. ${ }^{29}$
Kurjak et al conducted a study by 4D ultrasound and confirmed earlier findings made by 2D ultrasonography, that there is behavioral pattern continuity from prenatal to postnatal life. ${ }^{29,30}$ Assessment of neonatal behavior is a better method for early detection of CP than neurological examination alone. ${ }^{22,30,31}$ In our work we observed that there were no movements observed in the fetuses which were not present in neonates. ${ }^{32}$ The most frequent were hand to mouth and hand to face fetal and neonatal movements. Hand to mouth and hand to face movements were more frequent in fetuses than in neonates, while all other hand movements were less frequent in neonates than in fetuses. ${ }^{29}$

In our systematic study of fetal behavior by $4 \mathrm{D}$ sonography we were able to observe different expressions and movements of fetal face, but the question was if they were indicating fetal awareness? ${ }^{33}$ Is it the facial expression of the fetus that can help in understanding what fetus in utero would like to communicate? As our recent investigation showed, there is a behavioral continuity from fetal to neonatal life, which probably includes facial expression. ${ }^{33}$ Possibilities of 4D sonography are demonstrating the prenatal onset of a brain damage, based on morphological and functional signs. There is no doubt that this observation will be helpful, even though that prenatally observed signs are not yet highly predictive due to the brain immaturity, their identification will be at least recognized as a retrospective marker for a prenatal insult. ${ }^{34}$

Are we approaching the era when there will be applicable neurological test for fetus and assessment of neonate will be just the continuation? ${ }^{33,34}$ This is still not easy question to answer, because even postnatally there are several neurological methods of evaluation, while in utero we are dealing with more complicated situation and less mature brain. ${ }^{33,34}$ Could neonatal assessment of neurologically impaired fetuses bring some new insights into their prenatal neurological status is still unclear and to be investigated $?^{34,35}$ New scoring system for prenatal neurological assessment of the fetus proposed by Kurjak et al will give some new possibilities to detect fetuses at high neurological risk, although it is obvious that dynamic and complicated process of functional CNS development is not easy to investigate. ${ }^{36}$

In our recently published paper concerning the assessment of fetal behavior in high-risk pregnancies, we observed that some of the prenatal conditions are probably temporarily affecting fetal neurological status (ventriculomegaly, syndrome of intraamniotic infection, thrombocytopenia, thrombophylia, polihydramnios, preeclampsia, IUGR, achondroplasia), having tendency for improvement in neurological status after birth. ${ }^{36,37}$ On the basis of our preliminary results, we can only speculate why this 
happened after delivery. ${ }^{37}$ It is known that birthing process is affecting neonates neurologically, but it seems like some fetuses got liberated after birth due to numerous intrauterine constraints. $^{37}$

Our study showed that the new test might be useful in standardization of neurobehavioral assessments. ${ }^{36}$ Furthermore, there is a potential for antenatal detection of serious neurological problems. ${ }^{36-38}$

Preterm birth and/or VLBW does have an effect on health-related quality of life at various age groups. ${ }^{36-40}$ The impact of low birth weight and gestational age is greatest during the younger years, but the influence also extends into adolescence and adulthood. From a practical perspective, we may be able to improve neurological outcome and the quality of life of preterm and/or VLBW preschool- and school-aged children by referring them as early as possible to rehabilitation professionals. ${ }^{40}$

\section{Influence of Environment on Fetal and Neonatal Behavior}

Data concerning the influence of the gravity on fetal motor development are contradictory. The concept that the fetus floats in a state of weightlessness cannot be applied to the whole pregnancy, and after the fetus is confined by the uterus, it is exposed to the force of gravity. ${ }^{41}$ The fetus is not in significant contact with the walls of the amniotic sac until the very end of pregnancy, and sensory input arising from antigravity activity is absent, which is similar to the conditions of microgravity. ${ }^{42}$ Certain level of mechanical stress is necessary for the physiological development of the fetus. Along with muscle activity, gravitational loading also causes this mechanical stress. ${ }^{42}$ Buoyant forces apparently decrease fetal weight and in this way they reduce the effect of gravitation on the musculoskeletal system. ${ }^{42}$ It was clearly visible that until 21st week of gestation the fetus is in a condition similar to neutral buoyancy with apparent weight around 5\%. ${ }^{43}$ After the 26th week, the fetus is, to a significant extent, exposed to mechanical stress that occurs due to gravitation forces and has 60 to $80 \%$ apparent weight. ${ }^{43}$ If fetal movements cause deformation of the amniotic sac and the uterus then the force required to overcome elastic tension of the walls additionally contributes to the total mechanical stress to which the fetus is exposed. ${ }^{43}$ Total force of mechanical stress affecting the fetus in microgravity would, for this reason, be less than in a $1 \mathrm{G}$ environment. ${ }^{43}$ The development of antigravity muscular control is critical to normal motor development during the first year of life. After birth the newborn is exposed to the $1 \mathrm{G}$ environment. Movement against gravity begins during the first month of life, and by 4 months of age increased flexion control balances the strong extensor muscle patterns. ${ }^{43}$ Adequate development of trunk flexion and extension is a prerequisite to the development of anterior and posterior pelvic tilting, lateral trunk flexion and trunk elongation. ${ }^{44}$ These components enable the child to develop weight shifting, which in turn stimulates righting and equilibrium responses. ${ }^{44,45}$

\section{CONCLUSION}

Concerning the continuity from fetus to neonate in terms of neurobehavior, it could be concluded that fetus and neonate are the same persons in different environment. While in the womb, fetus is protected from the gravity which is not so important for its neurodevelopment, postnatally the neonate is exposed to the gravity during the labor and from the first moments of autonomous life. Development of motor control is highly dependent on antigravity forces enabling erect posture of infant or young child. These environmental differences should be kept on mind during prenatal as well as postnatal assessment.

\section{REFERENCES}

1. Schacher S. Determination and differentiation in the development of the nervous system. In: Kandel ER, Schwartz JH (Eds). Principles of neural science (2nd ed). New York, Amsterdam, Oxford: Elsevier Science Publishing 1985:730-32.

2. Kostovic I. Prenatal development of nucleus basalis complex and related fibre system in man: A hystochemical study. Neuroscience 1986;17:1047-77.

3. Kostovic I. Zentralnervensystem. In: Hinrichsen KV (Ed). Humanembryologie. Berlin: Springer-Verlag 1990:381-448.

4. Kuo AD. The relative roles of feedforward and feedback in the control of rhythmic movements. Motor Control 2002;6:129-45.

5. Ferrari F, Cioni G, Einspieler Ch, Roversi FM, Bos AF, Paolicelli $\mathrm{PB}$, et al. Cramped synchronized general movements in preterm infants as an early marker for cerebral palsy. Arch Pediatr Adolesc Med 2002;156:460-67.

6. Gairdner D, Pearson J. A growth chart for premature and other infants. Arch Dis Child 1971;46:783-87.

7. Eidelman AI. The living fetus - dilemmas in treatment at the edge of viability. In: Blazer S, Zimmer EZ (Eds): The embryoscientific discovery and medical ethics. Basel, Karger, 2005: 351-70.

8. Herculano-Houzel S. The human brain in nubers: A linearly scaled-up primate brain. Frontiers in Human Neuroscience 2009;3:1-11.

9. Zhang K, Sejnowski TJ. A universal scaling law between gray matter and white matter of cerebral cortex. PNAS 2000;97: 5621-26.

10. Kapellou O, Counsell SJ, Kennea N, Dyet L, Saeed N, Stark J, Maalouf E, et al. Abnormal cortical development after premature birth shown by altered allometric scaling of brain growth. PLoS Med 2006;3:1382-90.

11. Huizinga CT, Oudejans CB, Steiner RA, Clifton DK, Delemarrevan de Waal HA. Effects of intrauterine and early postnatal 
growth restriction on hypothalamic somatostatin gene expression in the rat. Pediatr Res 2000;48:815-20.

12. Lampl M, Gotsch F, Kusanovic JP, Espinoza J, GonÇalves L, Gomez R, et al. Downward percentile crossing as an indicator of an adverse prenatal environment. Annals of Human Biology 2008;35:462-74.

13. Latal-Hajnal B, Siebenthal K, Kovari H, Bucher HU, Largo RH. Postnatal growth in VLBW infants: Significant association with neurodevelopmental outcome. J Pediatr 2003;143:163-70.

14. Himmelmann K, Hagberg G, Wiklund LM, Eek MN, Uvebrant P. Dyskinetic cerebral palsy: A population-based study of children born between 1991 and 1998. Dev Med Child Neurol 2007;49:246-51.

15. Palmer FB. Strategies for the early diagnosis of cerebral palsy. J Pediatr 2004;145:S8-11.

16. Moster D, Wilcox AJ, Vollset SE, Markestad T, Lie RT. Cerebral palsy among term and post-term births. JAMA 2010;304: 976-82.

17. Cooke RWI, Foulder-Hughes L. Growth impairment in the very preterm and cognitive and motor performance at 7 years. Arch Dis Child 2003;88:482-87.

18. Jarvis S, Glinianaia SV, Blair E. Cerebral palsy and intrauterine growth. Clin Perinatol 2006;33:285-300.

19. Beckung E, Hagberg G, Uldall P, Cans C. Probability of walking in children with cerebral palsy in Europe. Pediatrics 2008;121: e187-92.

20. Paneth N. Establishing the diagnosis of cerebral palsy. Clin Obstet Gynecol 2008;51:742-48.

21. Krägeloh-Mann I, Cans C. Cerebral palsy update. Brain Dev 2009;31;537-44.

22. Kurjak A, Predojevic M, Stanojevic M, Salihagic-Kadic A, Miskovic B, Badrelden A, et al. Intrauterine growth restriction and cerebral palsy. Acta Informatica Medica 2010;18:64-82.

23. Inder TE, Warfield SK, Wang H, Hüppi PS, Volpe JJ. Abnormal cerebral structure is present at term in premature infants. Pediatrics 2005;115:286-94.

24. Thompson DK, Warfield SK, Carlin JB, Pavlovic M, Wang HX, Bear M, et al. Perinatal risk factors altering regional brain structure in the preterm infant. Brain 2007;130:667-77.

25. Gale CR, O’Callaghan FJ, Bredow M, Martyn CN. Avon longitudinal study of parents and children study team. The influence of head growth in fetal life, infancy and childhood on intelligence at the ages of 4 and 8 years. Pediatrics 2006; 118:1486-92.

26. Farooqi A, Hägglöf B, Sedin G, Gothefors L, Serenius F. Growth in 10 to 12-year-old children born at 23 to 25 weeks' gestation in the1990s: A Swedish National prospective follow-up study. Pediatrics 2006;118;e1452-65.

27. Einspieler C, Prechtl HFR, Bos AF, Ferrari F, Cioni G. Prechtl's method on the qualitative assessment of general movements in preterm, term and young infants. Mac Keith Press, Cambridge 2004.

28. Hadders-Algra M. General movements: A window for early identification of children at high risk for developmental disorders. J Pediatr 2004;145: S12-18.

29. Kurjak A, Azumendi G, Vecek N, et al. Fetal hand and facial expression in normal pregnancy studied by four-dimensional sonography. J Perinat Med 2003;31:496-508.

30. Kurjak A, Azumendi G. The fetus in three-dimension. Imaging, embryology and fetoscopy. Informa Healthcare, London 2007.
31. Kurjak A, Stanojevic M, Andonotopo W, Salihagic-Kadic A, Carera JM, Azumendi G. Behavioral pattern continuity from prenatal to postnatal life: A study by four-dimensional (4D) ultrasonography. J Perinat Med 2004;32:346-53.

32. Stanojevic M, Perlman M, Andonotopo W, Kurjak A. From fetal to neonatal behavioral status. Ultrasound Rev Obstet Gynecol 2004;4:459-71.

33. Kurjak A, Stanojevic M, Azumendi G, Carrera JM. The potential of four-dimensional ultrasonography in the assessment of fetal awareness. J Perinat Med 2005;33:46-53.

34. Kurjak A, Abo-Yaqoub S, Stanojevic M, Yigiter AB, Vasilj O, Lebit $\mathrm{D}$, et al. The potential of 4D sonography in the assessment of fetal neurobehavior-multicentric study in high-risk pregnancies. J Perinat Med 2010;38:77-82.

35. Kurjak A, Miskovic B, Stanojevic M, Amiel-Tison C, Ahmed B, Azumendi G, et al. New scoring system for fetal neurobehavior assessed by three- and four-dimensional sonography J Perinat Med 2008;36:73-81.

36. Stanojevic M, Talic A, Miskovic B, Vasilj O, Naim Shaddad A, Ahmed B, et al. An attempt to standardize Kurjak's antenatal neurodevelopmental test: Osaka consensus statement. Donald School Journal of Ultrasound in Obstetrics and Gynecology 2011;5:317-29.

37. Talic A, Kurjak A, Ahmed B, Stanojevic M, Predojevic M, Kadic AS, Di Renzo GC.The potential of 4D sonography in the assessment of fetal behavior in high-risk pregnancies. J Matern Fetal Neonatal Med 2011;24:948-54.

38. Predojević M, Stanojević M, Vasilj O, Kadić AS. Prenatal and postnatal neurological evaluation of a fetus and newborn from pregnancy complicated with IUGR and fetal hypoxemia. J Matern Fetal Neonatal Med 2011;24(5):764-67.

39. Kurjak A, Predojevic M, Stanojevic M, Talic A, Honemeyer U, Salihagic-Kadic A. The use of 4D imaging in the behavioral assessment of high fetuses. Imaging Med 2011;3(5):557-69.

40. Glennis Zwicker J, Susan Richardson Harris S. Quality of life of formerly preterm and very low birth weight infants from preschool age to adulthood: A systematic review. Pediatrics 2008;121;e366-76.

41. Bradley RM, Mistretta CM. Fetal sensory receptors. Physiol Rev 1975;55:352-82.

42. Elliott GB, Elliott KA. Some pathological, radiological and clinical implications of the precocious development of the human ear. Laryngoscope 1964;74:1160-71.

43. Sekulic SR, Lukac DD, Naumovic NM. The fetus cannot exercise like an astronaut: Gravity loading is necessary for the physiological development during second half of pregnancy. Med Hypotheses 2005;64:221-28.

44. Assaiante C, Mallau S, Viel S, Jover M, Schmitz C. Development of postural control in healthy children: A functional approach. Neural Plast 2005;12:109-18.

45. Sellers JS. Relationship between antigravity control and postural control in young children. Phys Ther 1988;68:486-90.

\section{ABOUT THE AUTHOR}

\section{Milan Stanojevic (Corresponding Author)}

Professor, Department of Obstetrics and Gynecology, Medical School University of Zagreb, Clinical Hospital 'Sveti Duh', Faculty of Health Science, Dubrovnik International University, Dubrovnik, Croatia e-mail: milan.stanojevic@optinet.hr 